\title{
Fatal intra-alveolar pulmonary bleeding complicating pulmonary embolectomy
}

\author{
A. R. M A KEY, B. P. BLISS, H. IKR A M, M . M. L. S U T CLIFFE, \\ and E. R. J. EMER Y \\ Department of Thoracic Surgery, Charing Cross Hospital, London W.C.2
}

Two patients are reported in whom fatal alveolar pulmonary haemorrhage occurred after pulmonary embolectomy. Possible causes and methods of prevention are discussed.

Pulmonary haemorrhage after embolic occlusion of a pulmonary artery usually follows the infarction of lung parenchyma. This complication is particularly prevalent in patients suffering from chronic congestive cardiac failure. The usual finding in such cases is obstruction of a segmental or smaller artery with infarction of the parenchyma supplied by it. The haemorrhage itself is usually slight and unimportant as regards eventual prognosis.

Parenchymal bleeding following obstruction of the main branches of the pulmonary artery, without overt evidence of infarction, and severe enough to cause death at a time when the obstructing embolus has been removed, is a rare event.

The purpose of this communication is to describe two patients in whom fatal pulmonary haemorrhage occurred following the removal of the obstructing clot. This complication of pulmonary embolectomy is relevant, as several nonoperative methods of treating acute pulmonary embolism have recently been introduced.

\section{CASE HISTORIES}

CASE 1 Mrs. R. S., aged 55 years, had a right partial nephrectomy for renal calculus. There was no previous history of cardiac disease. On the fifth postoperative day thrombophlebitis occurred in the left leg. A few hours later chest pain, dyspnoea, and a fall in blood pressure occurred. Heparin therapy was started, but 22 hours later there was a further episode with dyspnoea, cyanosis, and hypotension. An electrocardiogram showed acute right heart strain (Fig. 1). A pulmonary angiogram (Fig. 2) showed a complete blockage of the right main pulmonary artery and a further block in the artery to the left lower lobe.

In view of continued deterioration despite routine medical treatment, emergency pulmonary embolec- tomy with cardiopulmonary bypass was carried out34 hours after the initial embolus. A large coiled clot was removed from the right main pulmonary artery, and smaller fragments from the left lower lobar artery. The total time on full bypass was $20-$ minutes. While weaning off bypass there was a mas-: sive exudation of haemorrhagic pulmonary oedemaㅇ fluid from the endoctracheal tube. This rapidly re-s sembled pure blood and appeared to come from the right lung, which looked congested and infarcted. Theo left lung became flooded, despite attempts to excludeop the right with a Carlen's tube. It proved impossible to oxygenate the arterial blood, and the patient diedo from cardiac arrest.

Necropsy showed a healthy heart and coronary? vessels. There was antemortem thrombus in the left? femoral vein. Fluid blood was present in the right bronchial tree. The right lung was soft and hae-0 morrhagic with antemortem thrombus in the smalles arteries, but the major pulmonary vessels were normal. Histological examination confirmed infarction of the parenchyma (Fig. 3). An incidental findingi was a recent right adrenal haemorrhage (Fox, 1969)

CASE 2 Mr. J. W., aged 53 years, had suffered from myelofibrosis for many years. A splenectomy was per $\rightarrow$ formed for massive splenomegaly. He had been on steroid medication for the haematological condition and the operation was performed under steroid cover. This operation was performed with no untoward effects or unusual bleeding.

On the fourth postoperative day he collapsed on his way to the lavatory. A clinical diagnosis of acute pulmonary embolism was made, and in view of th $€$ inability to sustain the circulation despite vasos pressors, emergency embolectomy was undertaken ${ }^{+}$ without further investigation.

Cardiopulmonary bypass was begun four hours after the collapse. Fragmented clot was present im the right pulmonary arterial tree. This was removed and the artery was closed. A few seconds after the heart was restarted haemorrhagic fluid poured front the trachea. This became almost pure blood. As 

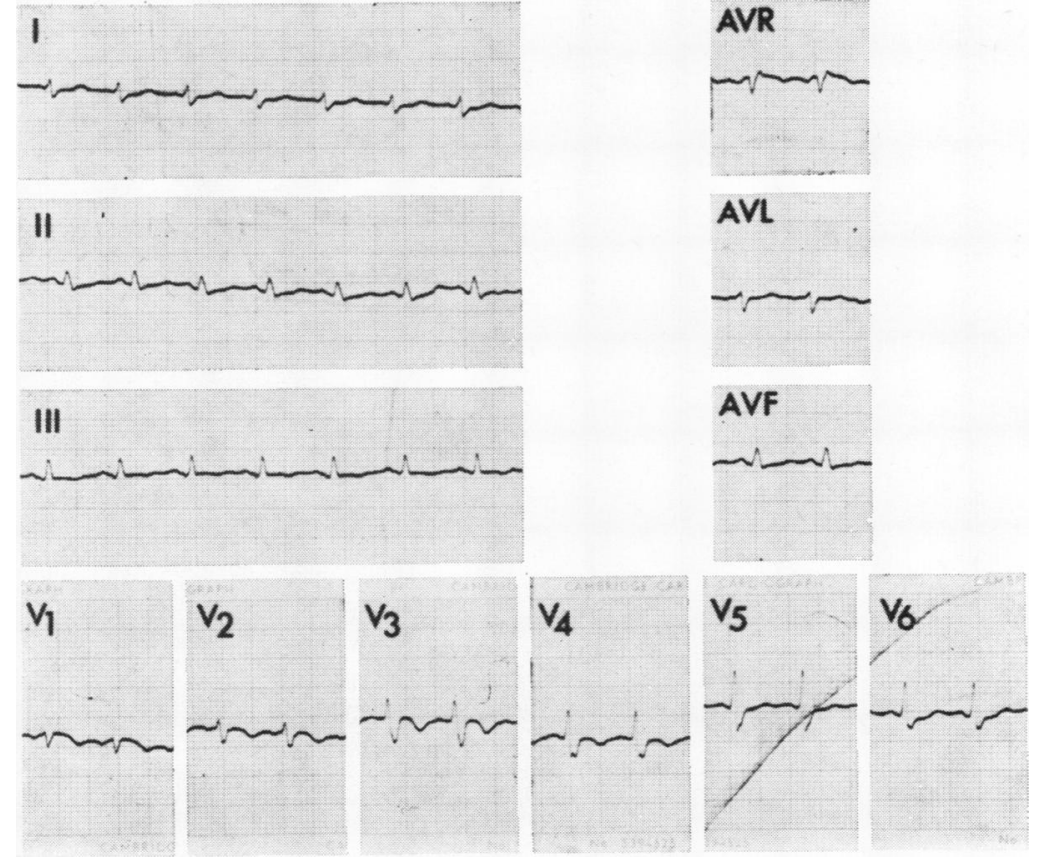

FIG. 1. Case 1. ECG shows right axis deviation and T-wave inversion in the right chest leads indicative of acute right heart strain.

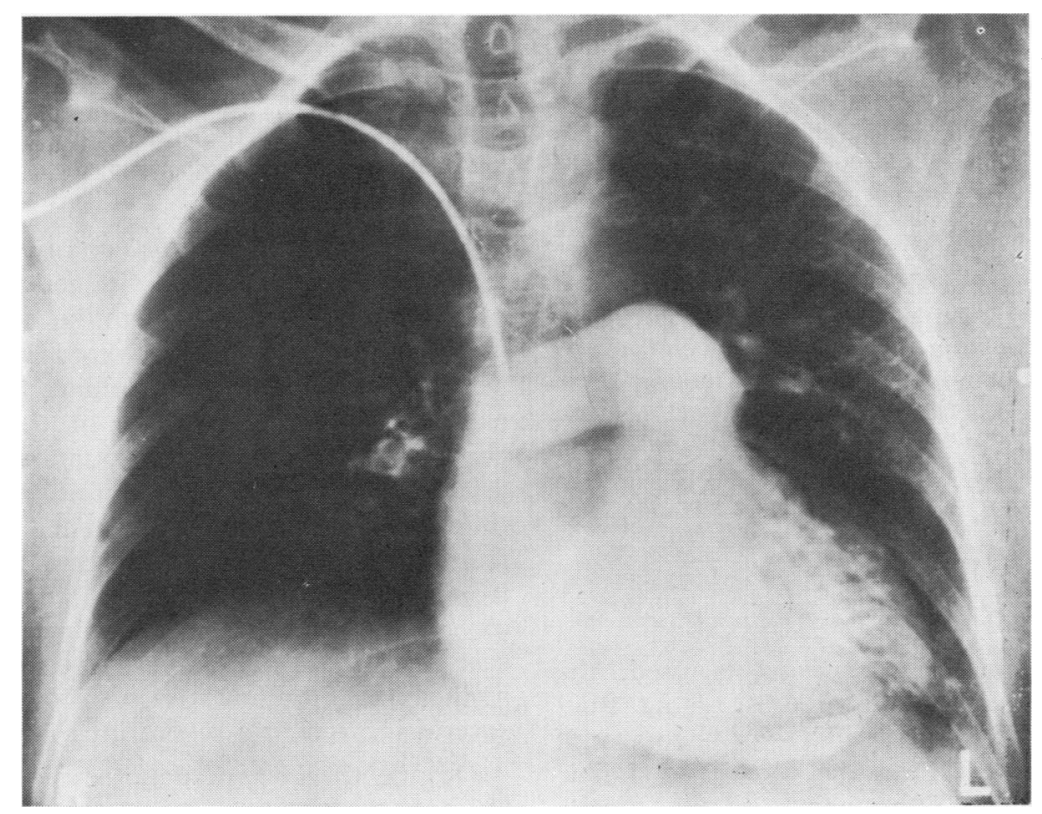

FIG. 2. Case 1. Angiocardiogram with injection of contrast into right atrium shows absence of filling of the pulmonary arterial tree on the right. 


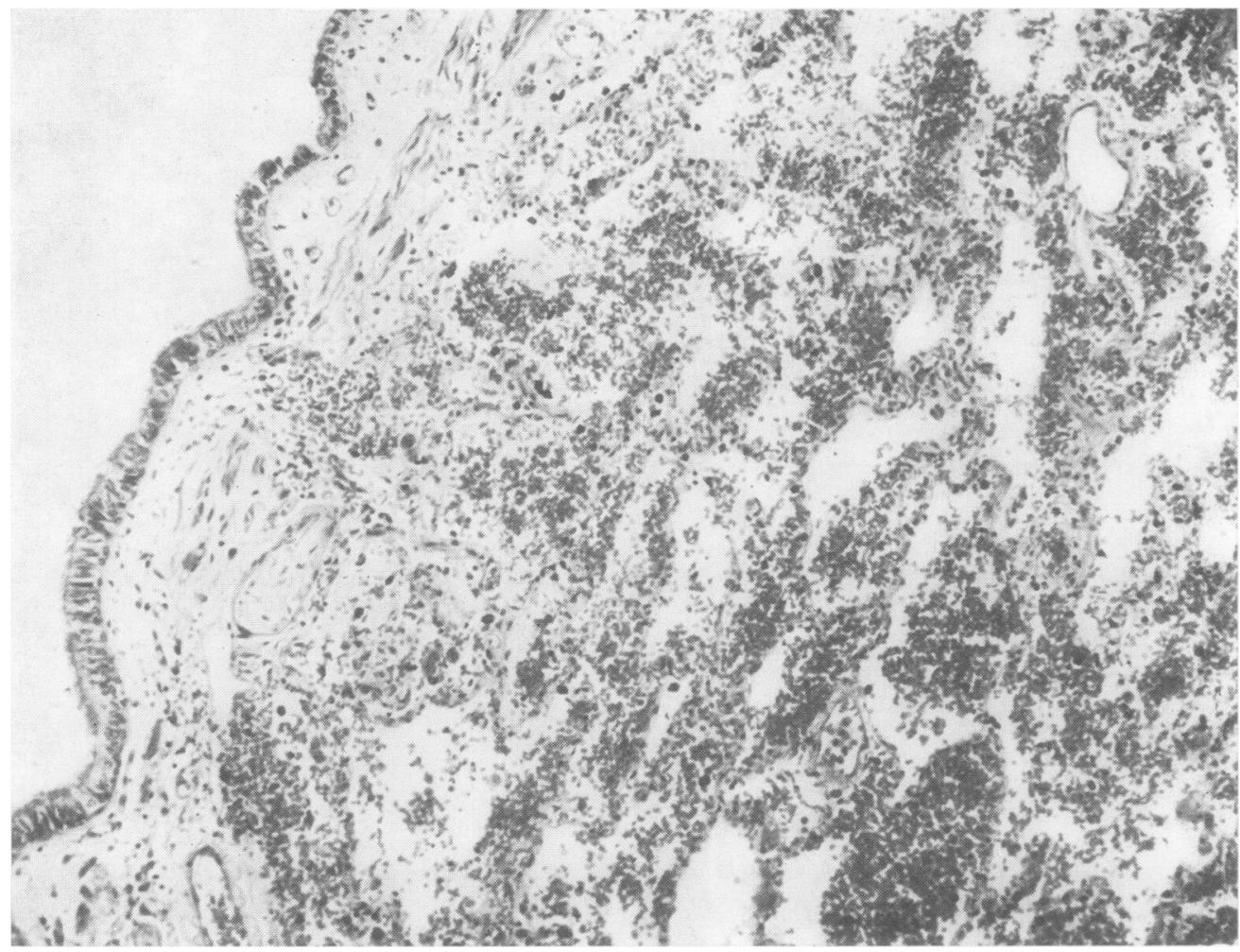

FIG. 3. Case 1. Section of lung shows severe intra-alveolar haemorrhage. $(\times 100)$.

Carlen's tube was passed, and this enabled the source of the bleeding to be identified as from the right main bronchus. The haemorrhage proved uncontrollable, and the patient died in asystolic cardiac arrest.

Gross anatomical examination after death showed a congested right lung, which appeared infarcted. Histological examination showed the alveoli to be filled with blood but no established infarction. There was disruption of the alveoli in some parts of the lung, and others showed areas of thickening and giant-cell infiltration. Intravascular thrombosis was present in the lung (Fig. 4). The adrenals were normal.

\section{DISCUSSION}

Although the term 'haemorrhage' is used, the exudate begins as a bloodstained oedema fluid and may not be pure blood. (No haematocrit values are available.) Thus the picture is similar to the haemorrhagic pulmonary oedema of the newborn (Adamson, Boyd, Normand, Reynolds, and Shaw, 1969), which occurs in conditions in which asphyxia is present, or likely to be present as well as having an association with raised left atrial pressure.

The incidence of this complication of pulmonary embolectomy as judged from a surveyo of the literature appears to be small. Only one identical case was discovered in the Englisho language literature (Castleman, 1964). In this patient, successful removal of the embolus hado been carried out and cardiopulmonary bypass was discontinued before the intrapulmonary haemor rhage began. Pulmonary oedema fluid welled upo from the tracheostomy tube and the patient died from hypoxia despite ventilation with $100 \%$ oxygen. Another point of similarity to our secondes case was that this patient had been on steroid medication preoperatively. Necropsy showedo several infarcts and severe intra-alveolar bleeding $\overrightarrow{\mathbb{D}}_{\overparen{R}}$

Despite the lack of detailed reports fatal intra- $\frac{\mathbb{Q}}{2}$ pulmonary bleeding in patients suffering fromo pulmonary embolism is mentioned. Stirling et al (1968) mention three patients who died withino 


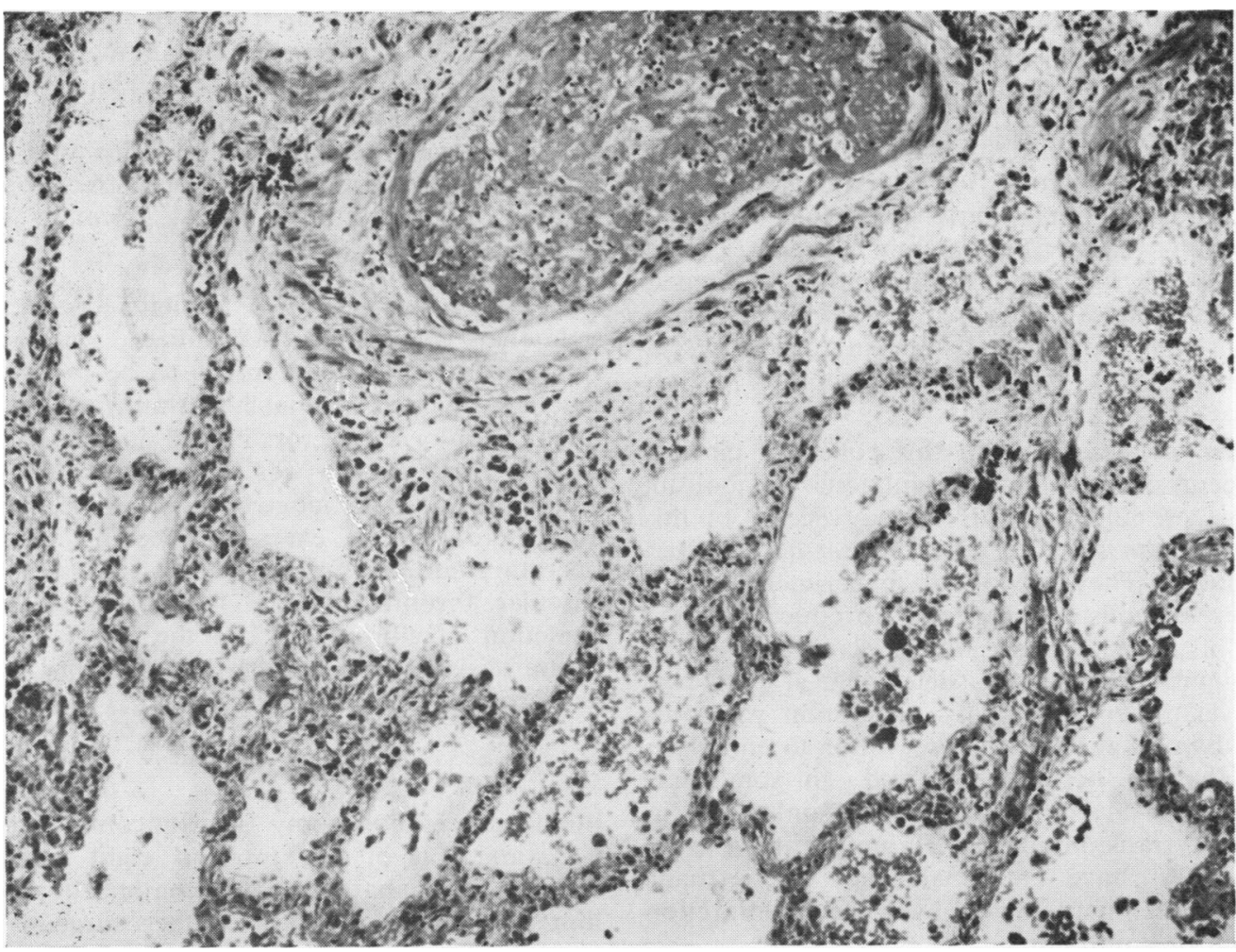

FIG. 4. Case 2. Section of lung shows some intra-alveolar haemorrhage and antemortem thrombus. $(\times 160)$.

three days after embolectomy from extensive intrapulmonary bleeding. Lam, Taber, and Gahagan (1967) report a patient who died of uncontrollable haemorrhage after embolectomy, but the site of bleeding is not mentioned. Hirsh et al. (1968), discussing streptokinase therapy for acute pulmonary embolism, attribute a falling haematocrit in some patients to intrapulmonary haemorrhage. However, Cross and Mowlem (1967) did not record this complication in a review of 137 pulmonary embolectomies, and hence its true incidence remains uncertain.

In our patients the clinical course and necropsy findings suggest that extensive damage to the lung vessels at capillary level was present, but any tendency to haemorrhage was prevented by the obstructing embolus. When this was removed, and pulmonary arterial blood flow was restored, the weakness in the capillary walls was revealed. In short, subclinical infarction had occurred and the restoration of blood flow converted it into a 'haemorrhagic' pulmonary infarction. Several studies have supported these observations (Agrest,
Lanari, and Roncoroni, 1953 ; Roach and Laufman, 1955).

HAEMODYNAMIC CONSIDERATION The infarction of 'lung tissue' in patients without previous cardiac or pulmonary pathology is difficult to explain. Virchow (1856) observed that obstruction of the branches of the pulmonary artery was not in itself sufficient to cause pulmonary infarction. Karsner and Ash (1912) demonstrated that infarction following experimental pulmonary embolism occurred only if congestion of the lung by ligation of the lobar veins or compression by pleural effusions had been produced previously. Other workers (Roach and Laufman, 1955 ; Chapman, Gugle, and Wheeler, 1949) have generally confirmed these findings. The only exception to the thesis that pre-existing derangement of lung circulation has to be present for pulmonary embolism to result in infarction is in the work of Steinberg and Mundy (1936), who produced infarcts in dogs using multiple lead shots as emboli.

Sufficient nutrition to maintain the integrity of 
the pulmonary tissues when massive embolic obstruction of lobar branches of the pulmonary arteries occurs would appear to come from anastomoses with the bronchial arteries. These anastomoses are normally present, and have been shown to increase following experimental ligation of a major pulmonary artery. In dogs, this collateral flow reaches $11 . / \mathrm{m}^{2} / \mathrm{min}$, roughly a third of the total right ventricular output (Bloomer, Harrison, Lindskog, and Liebow (1949). Good bronchial flow to infarcted areas in dogs is present within six days, reaching a maximum at four weeks (Steinberg and Mundy, 1936).

When interference with this collateral circulation occurs concomitant with pulmonary embolism, necrosis of pulmonary tissue may occur. In this connexion the work of Chapman et al. (1949) is interesting. They produced experimental pulmonary embolism using clots produced in the veins of normal dogs. Neither large nor small emboli produced infarcts detectable at necropsy. However, when pulmonary congestion was produced by alphanapthylthiourea prior to embolism then alveolar necrosis occurred. In some this consisted of alveolar haemorrhage only, and in others of frank infarction. Hampton and Castleman (1940) have called alveolar haemorrhage without other pathological evidence of infarction 'incomplete infarction'.

Systemic hypotension, when present, is dangerous for two reasons. First, the impairment of coronary flow may cause left ventricular failure and, secondly, reduction of bronchial flow may exacerbate the existing pulmonary ischaemia. Systemic hypotension requires treatment with pressor agents or with partial bypass to assist the circulation.

Elevation of pressure or distension of the left side of the heart due to 'heart failure' or to increased pulmonary venous return following relief of obstruction might result in capillary damage. This could be prevented by venting the left side.

Steroid function A feature common to our cases and to the one from the Massachusetts General Hospital (Castleman, 1964) is some abnormality of factors governing corticosteroid production. Case $1 \mathrm{had}$ an undiscovered haemorrhage in the right adrenal ; case 2 had been on chronic steroid medication, as had the case reported by Castleman. We are unable to implicate a definite mechanism. Steroids have an influence on thrombogenesis, and in Castleman's case every attempt to discontinue steroids was associated with a recurrence of chest pain. It is possible that $\overrightarrow{\vec{s}}$ steroid medication resulted in an increased pro- $\overline{0}$ pensity to thrombose the smaller lung vessels. $\frac{0}{0}$ Patients who are not on treatment with steroids $\overline{\bar{\alpha}}$ but whose acute pulmonary embolus was other- $\mathbb{D}$ wise managed identically have been free from this complication (Hampton and Castleman, $1940 ;$ is Makey and Bliss, 1966).

Coagulation defects All patients on cardio- $\vec{\omega}$ pulmonary bypass are heparinized, yet haenorrhage from the lungs appears to be rare, and it $\times$ would seem unlikely that heparinization can be a $\mathcal{N}$ major aetiological factor. Indeed, it is known that in case 1 all heparin was neutralized by the end $\vec{\circ}$ of the operation, as judged by protamine titration, and there was no excessive bleeding from the operation site. It is possible that extensive intra- $\overrightarrow{\vec{c}}$ vascular thrombosis (Fig. 4) may have led to depletion of fibrinogen, but the normal wound haemostasis and the clotting in laboratory investigations does not support this.

\section{CONCLUSIONS}

Pulmonary embolectomy is an uncommon operation, and out of our total of eight cases two $\mathbb{Q}$ showed this apparently rare complication. As is $\underset{F}{\vec{F}}$ hinted at in the literature, other surgeons may $\frac{0}{3}$ have experienced but not recorded it. A rise in left atrial pressure was not considered to be responsible for the bleeding, as both patients were still on partial bypass at the time of its occurrence. Left atrial pressure monitoring is not regularly performed in this emergency.

Apart from steroid medication or adrenal pathology, there does not appear to be any common factor by which these patients may be identified. The occurrence of this complication 0 unexpectedly at the end of an otherwise successful and uncomplicated pulmonary embolectomy, and o the fatal outcome in both cases, influence opinion in the direction of non-operative management. $\sigma$ Several methods are now available, including the $N$ use of streptokinase, urokinase and the venom of the Malayan pit viper, Ancrod. Since 1968 almost $\omega$ all our cases have been treated with streptokinase through the pulmonary artery by catheter used $\stackrel{0}{=}$ for angiography. Our results have been so satis- $\stackrel{\Phi}{\rightarrow}$ factory that the practical conclusion to be drawn from our experience is that the management of 0 major pulmonary embolism should be by fibrino- $\overrightarrow{\mathbb{D}}$ lysins, provided the patient is likely to live long $\underset{\mathbb{D}}{\stackrel{8}{*}}$ enough for these to take effect. If inadequate $\varrho$ improvement occurs, embolectomy can be performed later. 
If operation is unavoidable, then adequate steroid replacement therapy should be instituted, systemic hypotension treated with vasopressors or supportive perfusion, and the distension of the left side of the heart avoided. If pulmonary haemorrhage occurs, a trial of cross-clamping the pulmonary artery and bronchus at the hilum on the more affected side may be performed as a desperate measure to prevent the other lung being swamped with the exudate. Should this succeed in controlling the haemorrhage, then pneumonectomy should be considered.

We wish to thank the Charing Cross Research Committee for financial assistance.

Hamid Ikram was in receipt of a Charing Cross Hospital Board of Governors Research Fellowship.

\section{REFERENCES}

Adamson, T. M., Boyd, R. D. H., Normand, I. C. S. Reynolds, E. O. R., and Shaw, J. L. (1969). Haemorrhagic pulmonary oedema ("massive pulmonary haemorrhage") in the newborn. Lancet, 1, 494.

Agrest, A., Lanari, A., and Roncoroni, A. J. (1953). Embolia pulmonar unilateral experimental cón torax cerrado. Medicina (B. Aires), 13, 51.

Bloomer, W. E., Harrison, W., Lindskog, G. E., and Liebow, A. A. (1949). Respiratory function and blood flow in the bronchial artery after ligation of the pulmonary artery. Amer. J. Physiol., 157, 317.

Castleman, B. (Editor) (1964). Case records of the Massachusetts General Hospital. No. 32-1964. New Engl. J. Med., 271, 40.
Chapman, D. W., Gugle, L. J., and Wheeler, P. W. (1949). Experimental pulmonary infarction-abnormal pulmonary circulation as a prerequisite for pulmonary infarction following an embolus. Arch. intern. Med., 83, 158.

Cross, F. S., and Mowlem, A. (1967). A survey of the current status of pulmonary embolectomy for massive pulmonary embolism. Circulation, 35, suppl. 1, p. 86.

Fox, B. (1969). Adrenal haemorrhage and necrosis resulting from abdominal operations. Lancet, 1, 600.

Hampton, A. O., and Castleman, B. (1940). Correlation of postmortem chest teleroentgenograms with autopsy findings, with special reference to pulmonary embolism and infarction. Amer. J. Roentgenol., 43, 305.

Hirsh, J., Hale, G. S., McDonald, I. G., McCarthy, R. A., and Pitt, A. (1968). Streptokinase therapy in acute major pulmonary embolism: effectiveness and problems. Brit. med. J., 4, 729.

Karsner, H. T., and Ash, J. E. (1912). Studies in infarction: 2. Experimental bland infarction of the lung. $J$. med. Res., 27, 205.

Lam, C. R., Taber, R. E., and Gahagan, T. (1967). Surgical measures for the prevention and treatment of pulmonary embolism. Pacif. Med. Surg., 75, 71.

Makey, A. R., and Bliss, B. P. (1966). Pulmonary embolectomy-a review of five cases with three survivals. Lancet, 2, 1155.

Roach, H. D., and Laufman, H. (1955). Relationship between pulmonary embolism and pulmonary infarction: an experimental study. Ann. Surg., 142, 82.

Steinberg, B., and Mundy, C. S. (1936). Experimental pulmonary embolism and infarction. Arch. Path., 22, 529.

Stirling, G. R., Morris, K. N., McLean, K. H., Mirams, J. A., Rubinstein, D., and Wagner, G. R. (1968). Surgical aspects of pulmonary embolism. Med. J. Aust., 1, 571.

Virchow, R. (1856). Thrombose und Embolie. Gesammelte Abhandlungen zur wissenschaftlichen Medizin, pp. 219-226. Meidinger, Frankfurt am Main. 